\title{
Improving Energy Efficiency of Cooperative Femtocell Networks via Base Station Switching Off
}

\author{
Woongsup Lee ${ }^{1}$ and Bang Chul Jung ${ }^{2}$ \\ ${ }^{1}$ Department of Information and Communication Engineering, Gyeongsang National University, Jinju, Republic of Korea \\ ${ }^{2}$ Department of Electronics Engineering, Chungnam National University, Daejeon, Republic of Korea \\ Correspondence should be addressed to Bang Chul Jung; bangchuljung@gmail.com
}

Received 26 August 2016; Accepted 16 October 2016

Academic Editor: Jung-Ryun Lee

Copyright (c) 2016 W. Lee and B. C. Jung. This is an open access article distributed under the Creative Commons Attribution License, which permits unrestricted use, distribution, and reproduction in any medium, provided the original work is properly cited.

Recently, energy efficiency (EE) of cellular networks has become an important performance metric, and several techniques have been proposed to increase the EE. Among them, turning off base stations (BSs) when not needed is considered as one of the most powerful techniques due to its simple operation and effectiveness. Herein, we propose a novel BS switching-off technique for cooperative femtocell networks where multiple femtocell BSs (FBSs) simultaneously send packets to the same mobile station (MS). Unlike conventional schemes, cooperative operation of FBSs, also known as coordinated multipoint (CoMP) transmission, is considered to determine which BSs are turned off in the proposed technique. We first formulate the optimization problem to find the optimal set of FBSs to be turned off. Then, we propose a suboptimal scheme operating in a distributed manner in order to reduce the computational complexity of the optimal scheme. The suboptimal scheme is based on throughput ratio (TR) which specifies the importance of a particular FBS for the cooperative transmission. Through simulations, we show that the energy consumption can be greatly reduced with the proposed technique, compared with conventional schemes. Moreover, we show that the suboptimal scheme also achieves the near-optimal performance even without the excessive computations.

\section{Introduction}

In recent days, environmental pollution has become one of the biggest threats to mankind. The generation of electricity is one of the major sources of environmental pollution due to the use of fossil fuel or nuclear energy [1]. Given that the amount of electricity consumed by telecommunication systems (TSs) is considerably huge, a lot of efforts have been made to reduce the electricity usage in TSs such as the use of renewable electricity generation in base station (BS) $[2,3]$. The TSs with less electricity usage, that is, green cellular networks (GCNs), will be more important in the near future because the amount of electricity consumption is expected to increases nearly $20 \%$ per year [4].

One way to reduce the electricity consumption by TSs is to improve the energy efficiency of TS. Particularly, it is crucial to increase the energy efficiency of BS, since it consumes much more electricity compared to other network entities (more than half of total electricity consumption in TSs is caused by BSs), for example, mobile stations (MSs). For this reason, various methods have been taken into account in order to improve the energy efficiency of BS $[5,6]$.

Most powerful yet simple way to improve the energy efficiency of BS is to halt the operation of redundant BSs. However, the set of BSs which are switched off should not be selected randomly but it should be carefully chosen because the shutdown of BSs can severely deteriorate the performance of cellular network (CN), and it can also cause the outage of mobile services. Therefore, the shutdown of BSs has recently been the subject of extensive investigation [3, 7-14].

First, in $[7,8]$, the shutdown of macro BSs was considered. Specifically, in [7], the shutdown of macro BSs which are located at rural area during night time, that is, the statistical shutdown scheme where network operators manually decide which BSs to be switched off, was considered and in [8] the energy efficiency and coverage probability of macro 


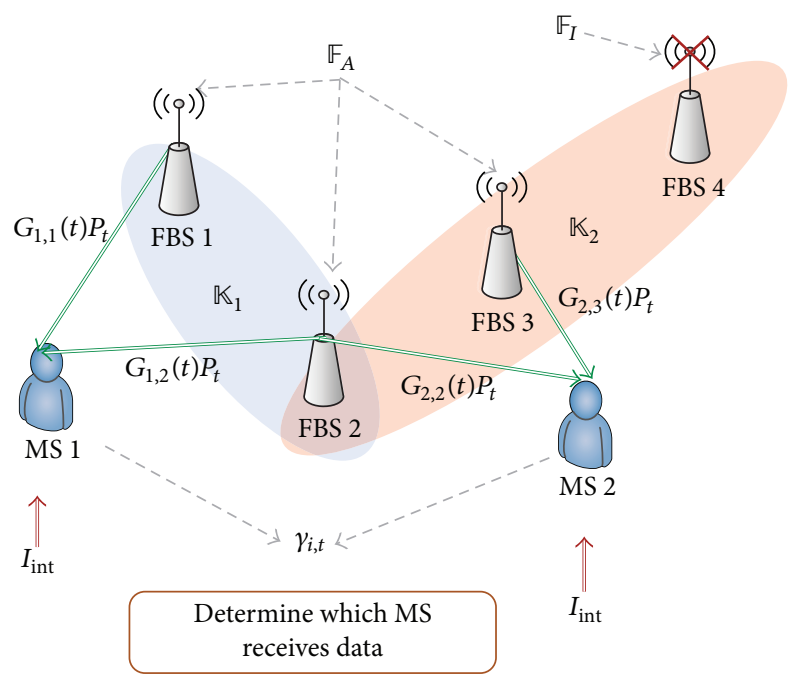

FIGURE 1: System model that shows MSs and FBSs utilizing cooperative transmission.

BS shutdown were investigated. However, the operation of macro BS is hard to be terminated in an adaptive way, according to real-time network condition, for example, traffic load, because the coverage of macro BS is wide such that the impact of switching off will be enormous. Accordingly in this paper we only take into account the shutdown of femto-BSs (FBSs) which have relatively small coverage area such that the impact of shutdown is rather limited to narrow area.

The shutdown of FBSs has been extensively investigated in recent days [9-13]. The author of [9] proposed the adaptive shutdown of indoor FBSs in which the operation of redundant FBSs is stopped by examining the achievable throughput. In [10], the operation of FBSs which are assigned small number of subcarriers was halted since the traffic load of these FBSs is low such that the shutdown of these FBSs is unlikely to deteriorate the performance of $\mathrm{CN}$ severely. Finally, the distance between the FBS and MSs is taken into account in the determination of the set of FBSs to shut down; that is, FBSs which are far from MSs are turned off since the contribution of these FBSs to the performance of $\mathrm{CN}$ is likely to be minor.

Usually, the traffic load on each FBS is used as a metric in determining FBSs to be shut down; that is, FBS with low traffic load is more likely to be turned off [8-10] because the traffic load indicates the importance of FBS in network. However, the use of traffic load can be inappropriate when multiple FBSs cooperate in data transmission; that is, cooperative transmission is considered, especially when coordinated multipoint (CoMP) $[15,16]$ is used. To be more specific, in the cooperative transmission, the incurred traffic load does not necessarily indicate the significance of FBSs in network.

For example, let us assume the scenario in which three FBSs (in Figure 1, FBS 4 is not participating in data transmission) (FBS 1, FBS 2, and FBS 3) are cooperatively transmitting data to two MSs (MS 1 and MS 2); compare Figure 1. Moreover, assume that FBS 1 and FBS 3 are close to MS 1 and MS 2, respectively, and FBS 2 is in between two FBSs such that FBS 1 and FBS 2 cooperatively transmit data to MS 1 and FBS 2 and FBS 3 cooperatively transmits data to MS 2 . Furthermore, assume that FBS 2 is farther away from MS 1 and MS 2 compared with FBS 1 and FBS 3 , respectively. In this scenario, although the traffic load of FBS 2 is larger than that of other two FBSs, it is more beneficial to turn off the FBS 2 because it unlikely deteriorates the throughput of network severely.

Therefore, new metric is needed in the determination of FBSs which utilize cooperative transmission. Given that FBSs are likely to cooperate with each other due to low transmit power, it is crucial to devise the efficient shutdown of FBS with cooperative transmission. Although [14] considered the shutdown of FBSs which use CoMP, FBSs which belong to the same cooperative transmission set cannot be selectively turned off, such that the network performance cannot be optimized. Moreover, the joint use of shutdown and CoMP was considered in [13]; however, BSs to be switched off are determined without taking into account the CoMP.

(A) Our Contributions. We herein propose an efficient shutdown scheme of FBSs considering cooperative transmission. The main contributions are as follows:

(1) In our proposed shutdown scheme, the operation of FBSs which uses cooperative transmission is efficiently halted with low computational complexity in distributed manner. Unlike previous approaches which mainly focus on the use of traffic load, we propose to use throughput ratio (TR) in determining the set of FBSs to be shut down such that the data rate of cooperative transmission is not overly degraded. To the best of our knowledge, this paper is the first study to use TR in the FBS shutdown.

(2) We investigate the performance of our proposed scheme using simulation. The results show that our proposed scheme achieves almost the same performance as the scheme based on the optimization problem, while the number of computations can be significantly reduced which makes our proposed scheme more practical. It is also shown that the power consumption of our proposed scheme is far below compared to that of the conventional scheme.

The remainder of the paper is organized as follows. In Section 2, we describe our system model and the optimization problem to find optimal set of FBSs to be turned off is formulated in Section 3. The proposed heuristic scheme is explained in Section 4 which is followed by the simulation results in Section 5. Finally, in Section 6, we provide our conclusions.

\section{System Model}

Our system model is depicted in Figure 1. In this paper, we consider the downlink of $\mathrm{CN}$ in which multiple FBSs cooperatively transmit the same data to MS on single channel, for example, CoMP $[15,16]$. We assume that multiple FBSs and MSs coexist. The sets of all FBSs, active FBSs, and inactive FBSs are denoted as $\mathbb{F}, \mathbb{F}_{A}$, and $\mathbb{F}_{I}$, respectively. For example, in Figure 1, FBS 1, FBS 2, and FBS 3 are in $\mathbb{F}_{A}$ while FBS 4 belongs to $\mathbb{F}_{I}$. 
We assume that the set of MSs is denoted as $\mathbb{M}$ and just represents the set of FBSs which can cooperate in data transmission to MS $i$; that is, not all FBSs are able to participate in data transmission to MSs. For example, in Figure 1, only FBS 1 and FBS 2 are in $\mathbb{K}_{1}$ and hence FBS 3 cannot transmit data to MS 1 . Note that a specific clustering algorithm to determine $\mathbb{K}_{i}$ is not taken into account in this work; that is, any clustering algorithm can be used. Moreover, we let $P_{t}$ be the transmit power of FBSs, where the transmit power control is not considered. Furthermore, the timevarying channel gain is denoted as $G_{i, j}(t)$, where $i$ is the index of MS, $j$ is the index of FBS, and $t$ is the time. It should be noted that $G_{i, j}(t)$ comprises both path-loss and fast fading. In addition, the bandwidth and the noise density are denoted as $W$ and $N_{0}$, respectively. In this paper, we assume that only one MS can receive data at the same time (this assumption is reasonable because FBSs within close proximity are unlikely to transmit data to multiple MSs simultaneously on the same channel, due to the excessive interference among them [15]), such that the interference among cooperative transmission is not considered. In addition, the amount of interference caused by other communication entities, for example, macro BS, is modeled as $I_{\text {int }}$; compare Figure 1. Finally, we assume that the power consumption of active FBS is $P_{A}>0$ and that of turned-off FBS is 0 .

We take into account a simple cooperative transmission scheme in which collaborating FBSs simply transmit the same signal to the target MS [15]. Then, the average throughput of $\mathrm{MS}_{i}, R_{i}\left(\mathbb{F}_{A}\right)$, can be formulated as follows:

$$
R_{i}\left(\mathbb{F}_{A}\right)=\mathbb{E}\left[\gamma_{i, t} W \log _{2}\left(1+\frac{\sum_{j \in\left\{\mathbb{F}_{A} \cap \mathbb{K}_{i}\right\}} G_{i, j}(t) P_{t}}{N_{0} W+I_{\text {int }}}\right)\right],
$$

where $\gamma_{i, t}$ is the indicator (given that multiple MSs cannot receive data at the same time in our system model, $\sum_{i=1}^{|\mathbb{M}|} \gamma_{i, t}=$ 1) which shows whether $\mathrm{MS}_{i}$ receives data at time $t$; that is, $\gamma_{i, t}=1$ when FBSs transmit data to $\mathrm{MS}_{i}$ at time $t$ and $\gamma_{i, t}=0$ otherwise. Moreover, $\mathbb{E}[\cdot]$ is the expectation. In (1), it is worth noting that only the signal power from active FBSs is accumulated.

Moreover, the average energy consumption of $\mathrm{CN}$ when the set of active FBSs is $\mathbb{F}_{A}$, which we denote as $E\left(\mathbb{F}_{A}\right)$, can be written as follows:

$$
E\left(\mathbb{F}_{A}\right)=P_{A}\left|\mathbb{F}_{A}\right|,
$$

where $|\cdot|$ denotes the cardinality of the set; for example, $|\mathrm{M}|$ corresponds to the number of all MSs. Note that when none of FBSs is turned off, the average throughput of $\mathrm{MS}_{i}$ and the average energy consumption of $\mathrm{CN}$ will become $\mathbb{E}\left[\gamma_{i, t} W \log _{2}\left(1+\left(\sum_{j \in \mathbb{K}_{i}} G_{i, j}(t) P_{t}\right) /\left(N_{0} W+I_{\text {int }}\right)\right)\right]$ and $P_{A}|\mathbb{F}|$, respectively, because $\mathbb{F}_{A}$ becomes $\mathbb{F}$.

Finally, we can derive the average traffic load of $\mathrm{FBS}_{j}$, when the active set of FBSs is $\mathbb{F}_{A}$, which we denote as $U_{j}\left(\mathbb{F}_{A}\right)$, as follows:

$$
U_{j}\left(\mathbb{F}_{A}\right)=\mathbb{E}\left[\sum_{i \in \mathbb{M}} R_{i, t}\left(\mathbb{F}_{A}\right) \mathbb{1}_{j \in \mathbb{K}_{i}} \mathbb{1}_{j \in \mathbb{F}_{A}}\right],
$$

where $\mathbb{1}(x)$ is the indicator function whose value is 1 when $x$ is true and 0 otherwise.

\section{Problem Formulation}

The optimization problem to find the optimal set of switchedoff FBSs can be formulated based on our system model. The objective of the formulated problem is the minimization of the total electricity consumption of FBSs, $E\left(\mathbb{F}_{\mathbb{A}}\right)$, while guaranteeing the performance degradation of $\mathrm{CN}$ to be less than the predefined threshold. To be more specific, we assume that the total network throughput should be larger than the predefined threshold, $R_{\mathrm{thr}}$. Then, the optimization problem to find $\mathbb{F}_{A}$ can be formulated as follows:

$$
\begin{aligned}
\underset{\mathbb{F}_{A} \subset \mathbb{F}}{\operatorname{minimize}} & E\left(\mathbb{F}_{\mathbb{A}}\right) \\
\text { s.t. } & \sum_{i \in \mathbb{M}} R_{i}\left(\mathbb{F}_{A}\right) \geq R_{\text {thr }} .
\end{aligned}
$$

It is worth noting that problem (4) is a binary programming such that it is nonconvex and the solution cannot be found within short computation time, especially, when the number of FBSs is large; that is, $|\mathbb{F}| \gg 0$. In order to solve this excessive computational complexity problem, we propose a heuristic way to find the optimal $\mathbb{F}_{A}$ within polynomial time in Section 4.

Note that the optimization problem to find $\mathbb{F}_{A}$ with conventional shutdown scheme, where FBSs with low traffic load are turned off, can be formulated as follows:

$$
\begin{aligned}
\underset{\mathbb{F}_{A} \subset \mathbb{F}}{\operatorname{minimize}} & E\left(\mathbb{F}_{\mathbb{A}}\right) \\
\text { s.t. } & \sum_{i \in \mathbb{M}} R_{i}\left(\mathbb{F}_{A}\right) \geq R_{\mathrm{thr}} \\
& U_{a}(\mathbb{F}) \geq U_{b}(\mathbb{F}), \quad \forall a \in \mathbb{F}_{\mathbb{A}}, b \in \mathbb{F}_{\square} .
\end{aligned}
$$

In (5), the last constraint guarantees the FBSs with low traffic load to be switched off. It should be noted that, due to this last constraint, the optimal solution of (5) can be found within polynomial time unlike optimization problem (4). To be more specific, the optimal solution can be found by turning off FBS in ascending order of traffic load until the constraint on data rate is satisfied. However, the solution will work poorly in practice when the cooperative transmission is considered, as shown in our performance evaluation.

\section{Proposed Scheme}

Herein, we have proposed a heuristic scheme based on TR, which indicates the contribution of certain FBS in the cooperative transmission, to solve the intractability problem of optimization problem (4). To be more specific, the TR value of $\mathrm{FBS}_{j}$, which we denote as $\mathrm{TR}_{j}$, can be defined as follows:

$$
\begin{aligned}
& \mathrm{TR}_{j} \\
& =\sum_{i \in \mathbb{M}} \frac{\mathbb{E}\left[\gamma_{i, t} W \log _{2}\left(1+G_{i, j}(t) P_{t} /\left(N_{0} W+I_{\mathrm{int}}\right)\right)\right]}{R_{i}(\mathbb{F})} .
\end{aligned}
$$

Note that, in (6), numerator corresponds to the expected achievable data rate of $\mathrm{MS}_{i}$ when $\mathrm{FBS}_{j}$ is the only FBS 


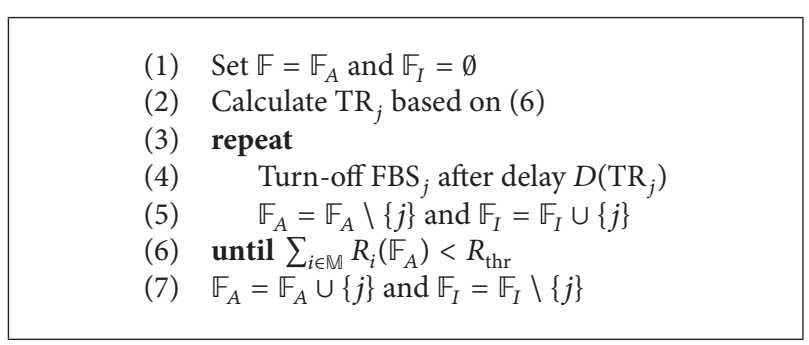

Algorithm 1: FBS shutdown based on TR.

to be activated. Accordingly, if the operation of $\mathrm{FBS}_{j}$ is important in view of cooperative transmission, the value of $\mathrm{TR}_{j}$ will be high. For example, if $\mathrm{FBS}_{j}$ is the only FBS which transmits data to $\mathrm{MS}_{i}$, then $\sum_{i \in \mathbb{M}}\left(\mathbb{E}\left[\gamma_{i, t} W \log _{2}(1+\right.\right.$ $\left.\left.\left.G_{i, j}(t) P_{t} /\left(N_{0} W+I_{\text {int }}\right)\right)\right] / R_{i}(\mathbb{F})\right)=1$; however, if $\mathrm{FBS}_{j}$ is not participating in transmission to $\mathrm{MS}_{i}$, that is, $i \notin \mathbb{K}_{i}$, then $\sum_{i \in \mathbb{M}}\left(\mathbb{E}\left[\gamma_{i, t} W \log _{2}\left(1+G_{i, j}(t) P_{t} /\left(N_{0} W+I_{\text {int }}\right)\right)\right] / R_{i}(\mathbb{F})\right)=0$.

In our proposed scheme, the TR value of FBSs is used to decide which FBS to be switched off, such that $\mathrm{FBS}_{j}$ with low $\mathrm{TR}_{j}$ will be turned off. More specifically, FBSs are switched off based on the ascending order of $\mathrm{TR}_{j}$. The shutdown of FBSs can be performed in distributed manner because each FBS can calculate its own $\mathrm{TR}_{j}$ based on its own channel condition and information from central management unit (CMU) which manages the operation of FBSs. Let $D(x)$ be the increasing function of $x$; for example, $D(x)=x^{2}$. Then, the operation of $\mathrm{FBS}_{j}$ can be halted after the delay of $D\left(\mathrm{TR}_{j}\right)$ and CMU can cease the shutdown of remaining active FBSs when $\sum_{i \in \mathbb{M}} R_{i}\left(\mathbb{F}_{A}\right)<R_{\text {thr }}$. The last turned-off FBS should be immediately turned on to guarantee our constraint. It is worth noting that the overhead of our proposed scheme will be negligible compared to that of the optimal scheme which can be found by directly solving (4). In summary, the overall procedures of the proposed algorithm are described in Algorithm 1.

\section{Performance Evaluation}

In this section, we investigate the performance of our proposed FBS shutdown scheme using simulation. In the performance evaluation, we assume that $N$ FBSs are randomly deployed and they transmit data using CoMP to two MSs which are located at the $(0,0)$ and $(50 \mathrm{~m}, 0)$; that is, $|\mathbb{M}|=2$. The distribution of FBSs is uniform, and $10 \mathrm{~m}<x<40 \mathrm{~m}$ and $-15 \mathrm{~m}<y<15 \mathrm{~m}$, where $(x, y)$ is the position of FBS; that is, FBSs are uniformly distributed within a square area. We assume that FBS whose value of $x$ is less than $30 \mathrm{~m}$ belongs to set $\mathbb{K}_{1}$ and FBS whose value of $x$ is larger than $20 \mathrm{~m}$ is within set $\mathbb{K}_{2}$, such that only FBSs whose $x$ are within $20 \mathrm{~m}<x<30 \mathrm{~m}$ can transmit data to both MSs (we have used this simulation scenario with only two MSs, in order to focus on the difference between conventional switching-off scheme and our proposed scheme; performance evaluation in more general environment, where more MSs coexist, will be an interesting extension of this work). IMT-R M.2135 urban macromodel (therefore, the path-loss becomes $34.5+$
TABLE 1: Simulation parameters.

\begin{tabular}{lc}
\hline Parameters & Values \\
\hline Number of MSs & 2 \\
Path-loss & $34.5+38 \log _{10}\left(d_{i, j}\right)$ (IMT-R M.2135 \\
& urban macromodel [17]) \\
$I_{\text {int }}$ & $-70 \mathrm{dBm}$ \\
$N_{0}$ & $-174 \mathrm{dBm} / \mathrm{Hz}$ \\
Bandwidth $(W)$ & $10 \mathrm{MHz}$ \\
$\gamma_{1, t}$ & 0.5 \\
$\gamma_{2, t}$ & 0.5 \\
Transmit power of FBS & $23 \mathrm{dBm}$ \\
\hline
\end{tabular}

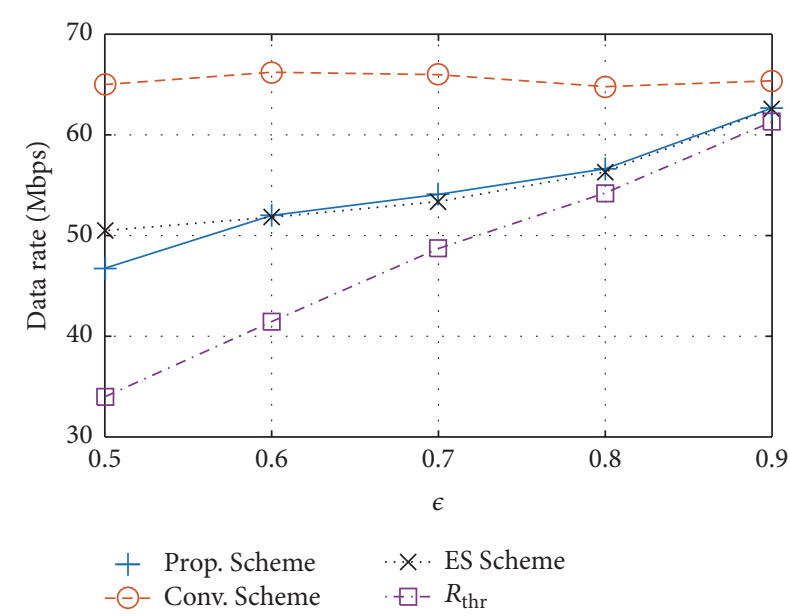

FIgURE 2: Data rate versus $\epsilon$ when $|\mathbb{F}|=10$.

$38 \log _{10}\left(d_{i, j}\right)$, where $d_{i, j}$ is the distance between $\mathrm{MS}_{i}$ and $\mathrm{FBS}_{j}$ ) is used for path-loss [17]. Furthermore, we assume that $I_{\text {int }}=-70 \mathrm{dBm}, N_{0}=-174 \mathrm{dBm} / \mathrm{Hz}, W$ is $10 \mathrm{MHz}$, $\gamma_{1, t}=\gamma_{2, t}=0.5$, and the transmit power of FBS, $P_{t}$, is $23 \mathrm{dBm}$. In addition, we assume that the channel experiences the fast fading which is modeled as a circularly symmetric complex Gaussian random variable with mean 0 and variance 1. Simulation parameters are summarized in Table 1.

In the performance evaluation, we examine the performance of our proposed scheme based on Algorithm 1 (Prop. Scheme) and also consider the performance of optimal scheme which can be found by solving (4) with exhaustive search (ES Scheme). Moreover, the conventional shutdown scheme, in which FBSs are switched off based on their traffic load, is also taken into account (Conv. Scheme), where FBS to be shut down is randomly chosen when multiple FBSs have the same traffic load. Note that the shutdown of Conv. Scheme is adjusted such that $\sum_{i \in \mathbb{M}} R_{i}\left(\mathbb{F}_{A}\right) \geq R_{\mathrm{thr}}$ is satisfied. Finally, in the simulation, we assume that $R_{\mathrm{thr}}=\epsilon \sum_{i \in \mathbb{M}} R_{i}(\mathbb{F})$, where $0 \leq \epsilon \leq 1$, such that $\epsilon$ indicates the degree of throughput deterioration that the system can tolerate. In other words, low value of $\epsilon$ means that decreasing power consumption is more important such that more FBSs will be turned off even if the throughput of the system is decreased significantly.

In Figures 2 and 3, the data rate and power consumption ratio, by varying $\epsilon$, are shown when the number of FBSs, $|\mathbb{F}|$, 


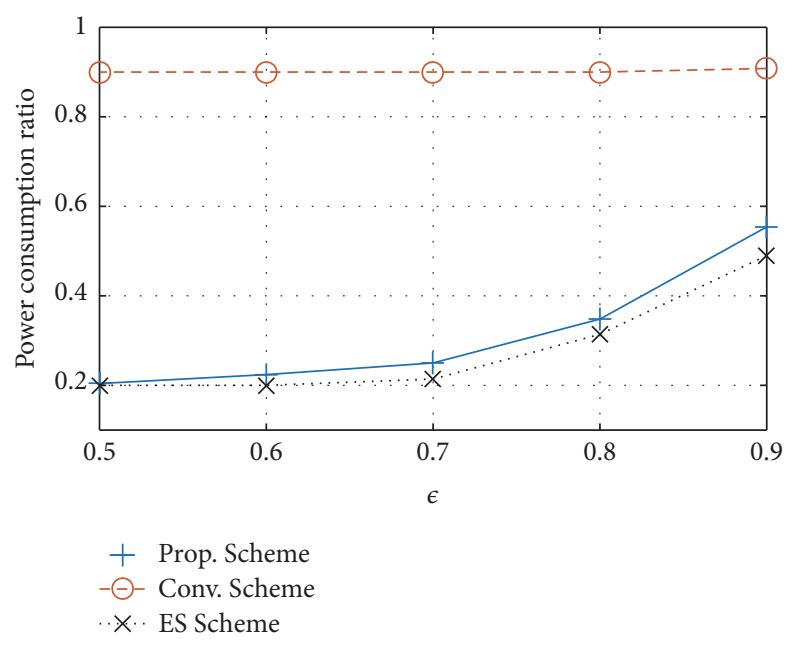

FIgURE 3: Power consumption ratio versus $\epsilon$ when $|\mathbb{F}|=10$.

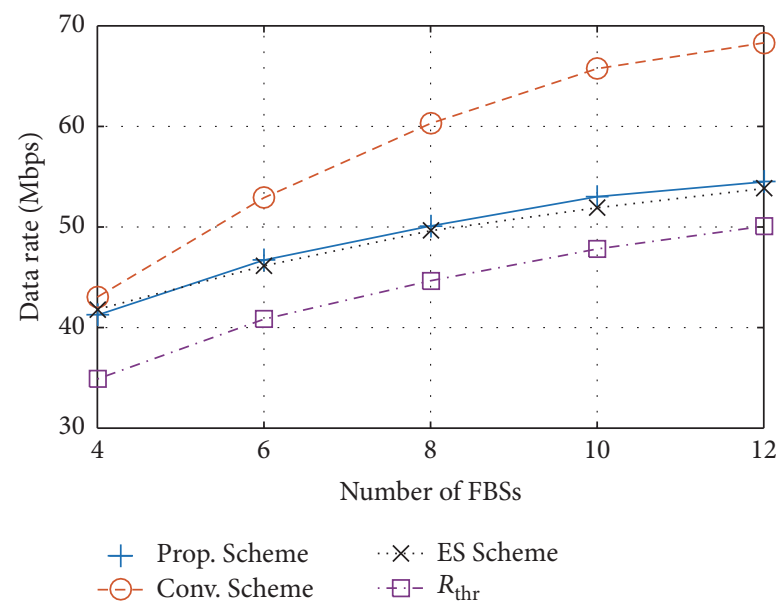

FIGURE 4: Data rate versus number of FBSs when $\epsilon=0.7$.

is 10 , where the power consumption ratio is the ratio of power consumption of the scheme and the power consumption without FBS shutdown, that is, $\left|\mathbb{F}_{A}\right| /|\mathbb{F}|$. For example, when the power consumption ratio is $0.5,50 \%$ of energy can be saved. We can find that the data rate of all considered schemes is larger than $R_{\mathrm{thr}}$; that is, constraints on data rate are satisfied in all cases. However, the data rate of Prop. Scheme and ES Scheme decreases as $\epsilon$ decreases while that of Conv. Scheme remains the same because the number of FBSs to be turned off is unchanged in Conv. Scheme while that of Prop. Scheme and ES Scheme decreases greatly, as can be verified from Figure 3. Specifically, in Prop. Scheme and ES Scheme, nearly $80 \%$ of FBSs can be turned off without violating the constraint on data rate such that energy consumption can be reduced significantly, which validates the benefit of our proposed scheme. Moreover, we can verify that our proposed scheme achieves near-optimal performance in terms of power consumption.

Figures 4 and 5 show the data rate and power consumption ratio by varying the number of FBSs when $\epsilon$ is 0.7 . We can observe that the data rate increases as the number

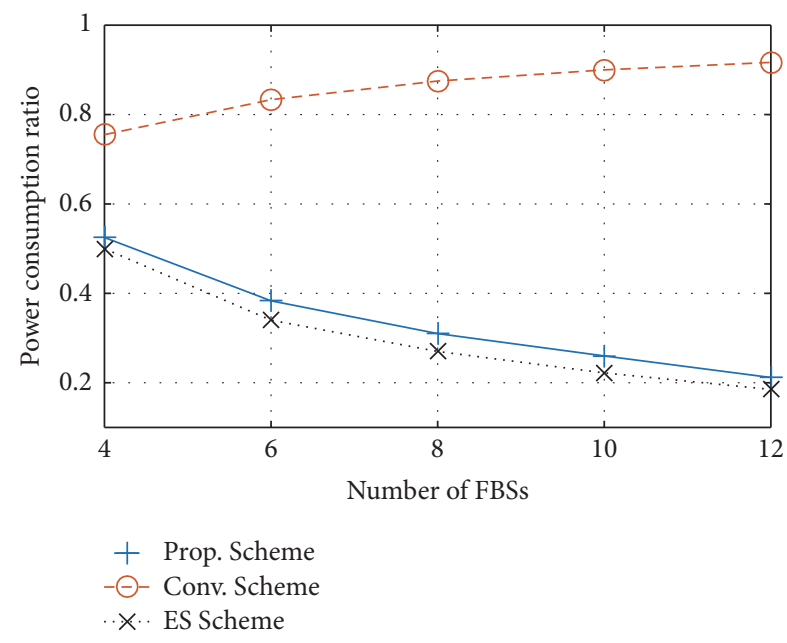

FIgURE 5: Power consumption ratio versus number of FBSs when $\epsilon$ $=0.7$.

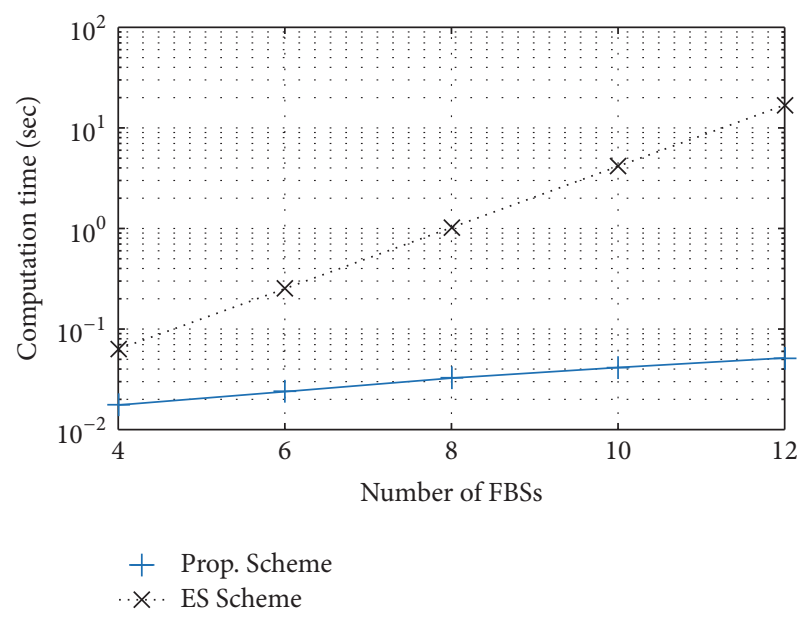

Figure 6: Computation time versus number of FBSs when $\epsilon=0.7$.

of FBSs increases because received signal power of MSs is likely to increase. It is worth noting that the data rate of all considered schemes exceeds $R_{\mathrm{thr}}$; that is, the constraint on data rate is satisfied even when $|\mathbb{F}|$ increases. Moreover, we can find that the power consumption ratio of our proposed scheme is much lower than that of Conv. Scheme, such that more energy can be saved in our proposed scheme compared to conventional FBS shutdown scheme. Particularly, we can observe that the gap between the power consumption ratio of our scheme and that of conventional scheme increases as $|\mathbb{F}|$ increases because the number of redundant FBSs is more when $|\mathbb{F}|$ is large; that is, the operation of more FBSs can be halted without hurting the data rate significantly. Therefore, we can conclude that our proposed scheme works better when the number of FBS is large. It should be noted that the power consumption ratio of Prop. Scheme and ES Scheme is almost the same; that is, the performance of our proposed scheme is nearly optimal, although Prop. Scheme is a heuristic scheme that works in distributed manner.

Finally, in Figure 6, the computation time of Prop. Scheme and ES Scheme by varying $|\mathbb{F}|$ is depicted. As can be 
seen from the results, the computation time of our proposed scheme is much lower than that of ES Scheme because the number of computations required for ES Scheme increases exponentially with $|\mathbb{F}|$. Therefore, our proposed scheme is much better to be implemented in practice, especially in large $\mathrm{CN}$ where large number of FBSs is considered.

\section{Conclusions}

In this paper, the efficient way to halt the operation of redundant FBSs in cooperative transmission was exploited. Given that existing FBS shutdown schemes are inappropriate for FBS with cooperative transmission, we proposed new scheme by formulating the optimization problem. In order to solve the excessive computational complexity problem, a heuristic scheme based on TR was devised, which can operate in distributed manner. Through simulations based on realistic parameters, it was shown that our proposed scheme achieves near-optimal performance without excessive computations, such that the power consumption of FBSs can be greatly reduced without overly degrading the data rate of $\mathrm{CN}$. The consideration of more generalized system model, in which FBSs transmit with different transmit power on multiple channels, might be an interesting extension of this work.

\section{Competing Interests}

The authors declare that they have no competing interests.

\section{Acknowledgments}

This research was supported by "Cooperative Research Program for Agriculture Science \& Technology Development (project title: Development of Swine Management Model with Animal-Metric for Livestock Welfare, Project no. PJ0105412015)," Rural Development Administration, Republic of Korea, and also supported by the Basic Science Research Program through the National Research Foundation of Korea (NRF) funded by the Ministry of Science, ICT \& Future Planning (MSIP) (NRF-2016R1A2B4014834). This work was also supported by Chungnam National University.

\section{References}

[1] X. Fang, S. Misra, G. Xue, and D. Yang, "Smart grid-the new and improved power grid: a survey," IEEE Communications Surveys \& Tutorials, vol. 14, no. 4, pp. 944-980, 2012.

[2] K. Kusakana and H. J. Vermaak, "Hybrid renewable power systems for mobile telephony base stations in developing countries," Renewable Energy, vol. 51, pp. 419-425, 2013.

[3] S. Zhang, N. Zhang, S. Zhou, J. Gong, Z. Niu, and X. Shen, "Energy-aware traffic offloading for green heterogeneous networks," IEEE Journal on Selected Areas in Communications, vol. 34, no. 5, pp. 1116-1129, 2016.

[4] A. Mukherjee, S. Bhattacherjee, S. Pal, and D. De, "Femtocell based green power consumption methods for mobile network," Computer Networks, vol. 57, no. 1, pp. 162-178, 2013.

[5] R. Mahapatra, Y. Nijsure, G. Kaddoum, N. Ul Hassan, and C. Yuen, "Energy efficiency tradeoff mechanism towards wireless green communication: a survey," IEEE Communications Surveys \& Tutorials, vol. 18, no. 1, pp. 686-705, 2016.

[6] F. K. Shaikh, S. Zeadally, and E. Exposito, "Enabling technologies for green internet of things," IEEE Systems Journal, 2015.

[7] E. Oh, B. Krishnamachari, X. Liu, and Z. Niu, "Toward dynamic energy-efficient operation of cellular network infrastructure," IEEE Communications Magazine, vol. 49, no. 6, pp. 56-61, 2011.

[8] Y. S. Soh, T. Q. S. Quek, and M. Kountouris, "Dynamic sleep mode strategies in energy efficient cellular networks," in Proceedings of the 2013 IEEE International Conference on Communications (ICC '13), pp. 3131-3136, Budapest, Hungary, June 2013.

[9] E. Yaacoub and A. Kadri, "Green operation of LTE-A femtocell networks benefiting from centralized control," in Proceedings of the 11th International Wireless Communications and Mobile Computing Conference (IWCMC '15), pp. 408-412, Dubrovnik, Croatia, August 2015.

[10] H. Nabuuma, E. Alsusa, and W. Pramudito, "A load-aware base station switch-off technique for enhanced energy efficiency and relatively identical outage probability," in Proceedings of the 81st IEEE Vehicular Technology Conference (VTC Spring '15), Glasgow, UK, May 2015.

[11] A. Bousia, A. Antonopoulos, L. Alonso, and C. Verikoukis, “'Green' distance-aware base station sleeping algorithm in LTEAdvanced," in Proceedings of the IEEE International Conference on Communications (ICC '12), pp. 1347-1351, Ottawa, Canada, June 2012.

[12] W. Vereecken, M. Deruyck, D. Colle et al., "Evaluation of the potential for energy saving in macrocell and femtocell networks using a heuristic introducing sleep modes in base stations," Eurasip Journal on Wireless Communications and Networking, vol. 2012, article 170, 2012.

[13] G. Cili, H. Yanikomeroglu, and F. R. Yu, "Cell switch off technique combined with coordinated multi-point (CoMP) transmission for energy efficiency in beyond-LTE cellular networks," in Proceedings of the 2012 IEEE International Conference on Communications (ICC '12), pp. 5931-5935, Ottawa, Canada, June 2012.

[14] A. Al-Dulaimi, A. Anpalagan, M. Bennis, and A. V. Vasilakos, " $5 \mathrm{G}$ green communications: C-RAN provisioning of CoMP and femtocells for power management," in Proceedings of the IEEE International Conference on Ubiquitous Wireless Broadband (ICUWB '15), pp. 1-5, Montreal, Canada, October 2015.

[15] W. Lee and D. Cho, "Simultaneous RTS and sequential CTS considering multiple cooperative relays," IEEE Transactions on Vehicular Technology, vol. 62, no. 5, pp. 2369-2374, 2013.

[16] W. Lee and H. Lee, "Performance evaluation of coordinated multi-point transmission and reception in indoor mobile communication systems," Journal of Information and Communication Convergence Engineering, vol. 11, no. 3, pp. 167-172, 2013.

[17] Report ITU-R M.2135-1, Guidelines for evaluation of radio interface technologies for IMT-Advanced, December 2009. 

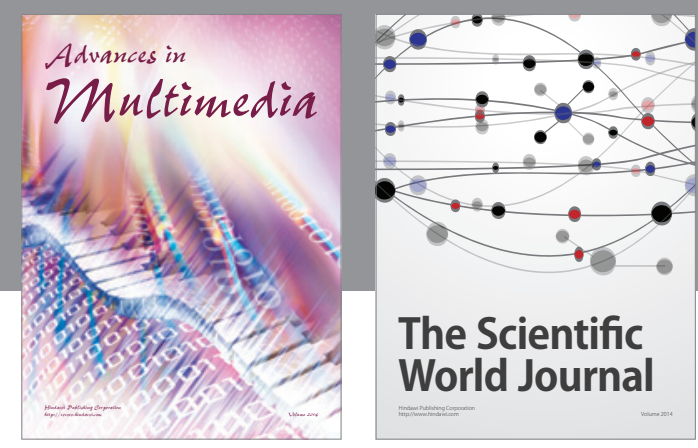

The Scientific World Journal
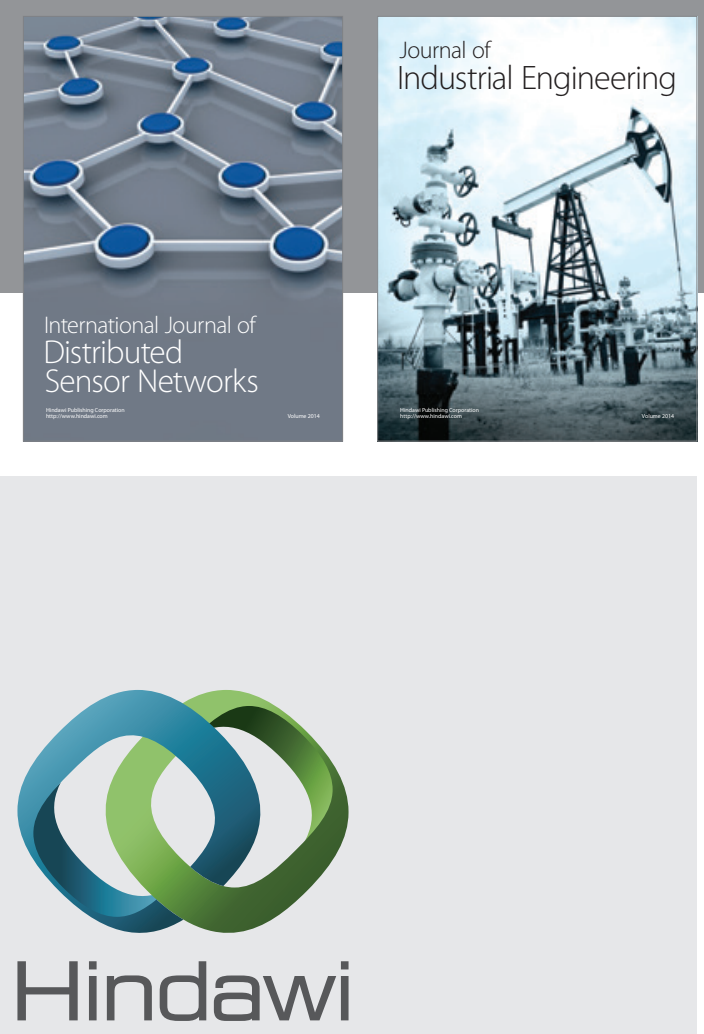

Submit your manuscripts at

http://www.hindawi.com

\section{Computer Networks} and Communications
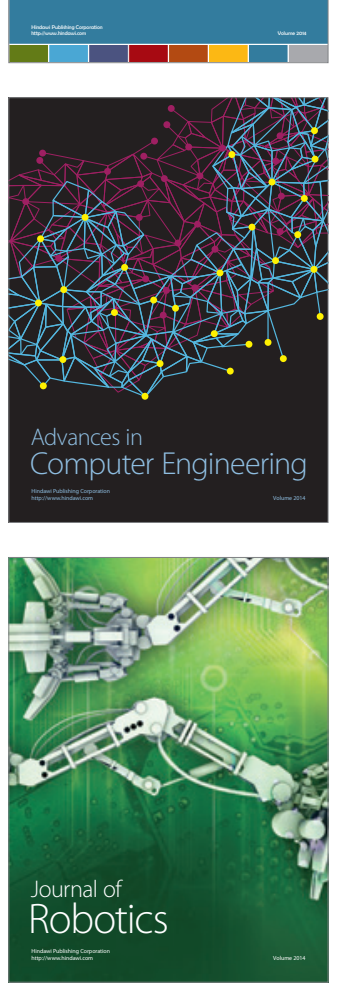
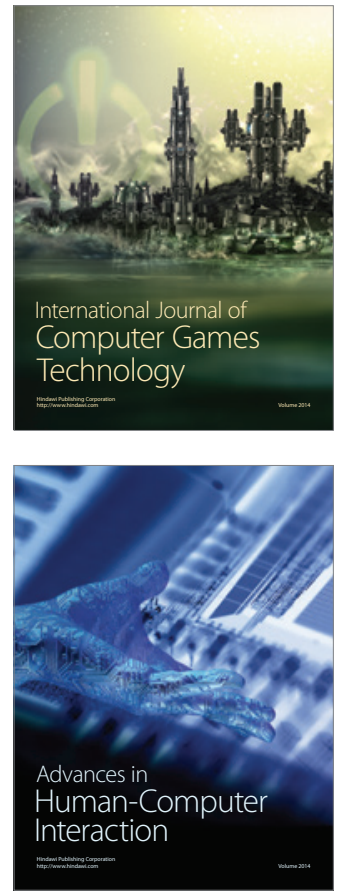
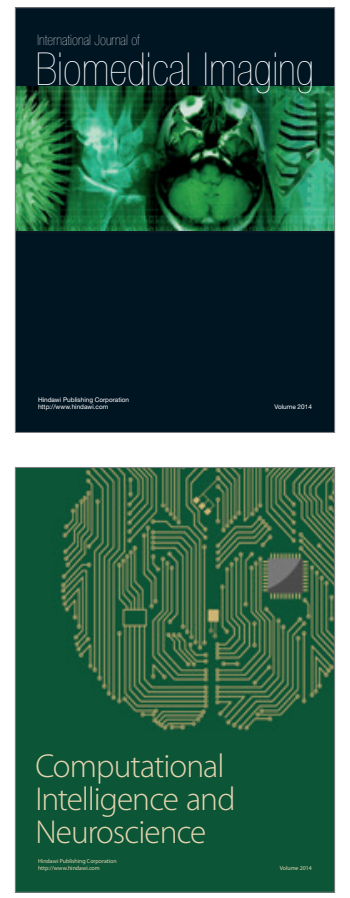
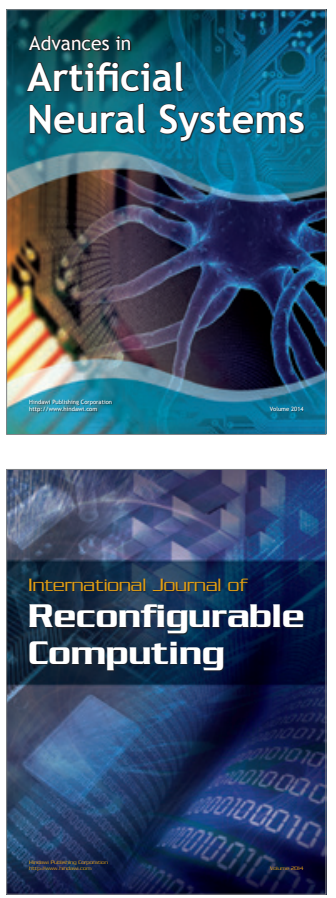
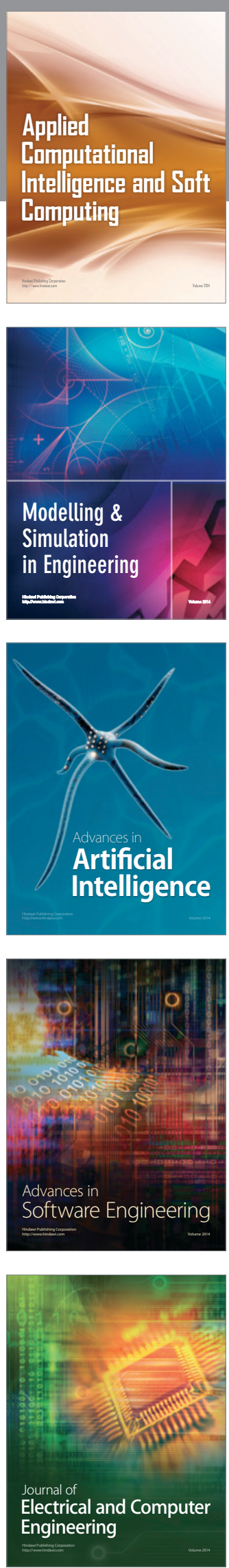